\title{
miR-27b inhibits gastric cancer metastasis by targeting NR2F2
}

\author{
Qingzhao Feng ${ }^{1 凶}$, Xionglin $\mathrm{Wu}^{5}$, Fuchao $\mathrm{Li}^{2}$, Beibei Ning ${ }^{4}$, Xiaofeng Lu ${ }^{1}$, Yin Zhang ${ }^{1}$, Ying Pan $^{3}$, \\ Wenxian Guan ${ }^{1 凶}$ \\ ${ }^{1}$ Department of General Surgery, The Affiliated Drum Tower Hospital of Medical School of Nanjing University, Nanjing 210008, \\ China \\ 2 Department of Gerontology, The Affiliated Drum Tower Hospital of Medical School of Nanjing University, Nanjing 210008, \\ China \\ ${ }^{3}$ Department of Orthopaedics, The Affiliated Drum Tower Hospital of Medical School of Nanjing University, Nanjing 210008, \\ China \\ 4 The Children's Hospital of Zhejiang University School of Medicine, Hangzhou 310003, China \\ ${ }^{5}$ Department of Emergency, The Yiwu Affiliated Hospital of Wenzhou Medical University, Yiwu 322000, China \\ $\triangle$ Correspondence: izaobao@vip.qq.com (Q. Feng), guan-wx@163.com (W. Guan)
}

Received June 26, 2016 Accepted October 20, 2016

\begin{abstract}
Increasing attention is focused on the down-regulation of miRNAs in cancer process. Nuclear receptor subfamily 2 (NR2F2, also known as COUP-TFII) is involved in the development of many types of cancers, but its role in gastric cancer remains elusive. In this experiment, oncomine and Kaplan-meier database revealed that NR2F2 was up-regulated in gastric cancer and that the high NR2F2 expression contributed to poor survival. MicroRNA-27b was targeted and down-regulated by NR2F2 in human gastric cancer tissues and cells. The ectopic expression of miR-27b inhibited gastric cancer cell proliferation and tumor growth in vitro and in vivo. Assays suggested that the overexpression of miR-27b could promote MGC-803 cells' migration and invasion and retard their metastasis to the liver. In addition, down-regulation of miR-27b enhanced GES-1 cells' proliferation and metastasis in vitro. These findings reveal that miR-27b is a tumor suppressor in gastric cancer and a biomarker for improving patients' survival.
\end{abstract}

Qingzhao Feng, Xionglin Wu, Fuchao Li contributed equally to this work.

Electronic supplementary material The online version of this article (doi:10.1007/s13238-016-0340-z) contains supplementary material, which is available to authorized users.
KEYWORDS miR-27b, NR2F2, gastric cancer, tumor metastasis

\section{INTRODUCTION}

Gastric cancer (GC), the second leading cause of cancerinduced death, has a high incidence in countries like China or Japan (Kang et al., 2014; Yan et al., 2015). Various innate and acquired factors, including $H$. pylori infection, genetic, epigenetic and molecular alterations, are involved in gastric tumorigenesis via their impact on genetic expression and signaling pathways (Milne et al., 2009). However, the mechanism of GC oncogenesis remains unknown. Nuclear receptor subfamily 2 (NR2F2, group $F$, member 2, also known as COUP-TFII or ARP1) is a key molecule in mesenchymal-epithelial interaction during organogenesis (Tsai and Tsai, 1997). Meanwhile, Kieback et al. find that NR2F2 is expressed in tumor cell lines of human endometrial (Kieback et al., 1996), pancreatic (Qin et al., 2010), colorectal (Shin et al., 2009), and breast cancers (Prahalad et al., 2010). The expression of NR2F2 in terminally differentiated epithelial cells functions in mesenchymal-endothelial interactions, angiogenesis, tumor growth and metastasis by inhibiting TGF- $\beta$-induced growth (Qin et al., 2013).

MicroRNAs (miRs), as small single-stranded noncoding RNAs, are key post-transcriptional negative regulators that completely or partially bind to complementary sites in the 3'untranslated-region ( $3^{\prime} U T R$ ) of target mRNAs. Recent studies show that miRNAs can regulate tumor growth, 
metastasis and progression (Ma et al., 2007; Ruan et al., 2009; Aleckovic and Kang, 2015). A single miRNA can downregulate the expression of multiple target genes and thereby inhibit tumor metastasis. Therefore, miRNAs can be targeted to modulate the invasion-metastasis cascade (Lin et al., 2015). MiR-27b is a strain of intronic miRNA that regulates chondrosarcoma (Huang et al., 2016), cervical carcinogenesis (Yao et al., 2016) and neuroblastom (Lee et al., 2012).

Interestingly, a recent report demonstrates that the lower level of miR-27b expression correlates with gastric cancer proliferation (Tao et al., 2015). However, little is known about the function of miR-27b in gastric cancer metastasis. In our study, miR-27b was downregulated in gastric cancer tissues, with an inverse correlation with lymph node metastasis. Meanwhile, the miR-27b overexpression inhibited the proliferation and invasion of gastric cancer cells in vitro and suppresses tumor growth and liver metastasis of gastric cancer cells in vivo. Conversely, miR-27b inhibitors significantly enhanced the proliferation and invasion of gastric cancer cell in vitro. Our study concludes that miR-27b plays a suppressive role in gastric cancer metastasis.

\section{RESULTS}

Survival time shortened by high NR2F2 expression in gastric cancer

The expression of NR2F2 was measured in gastric cancer patients by oncomine database. NR2F2 was significantly upregulated in gastric cancer tissues compared with normal tissues (Fig. 1A). Q-RT found that NR2F2 expression was significantly higher in gastric cancer tissues than in normal tissues (Fig. 1B). Analysis of immunohistochemical staining and Western blot also support this result (Fig. S1). We subsequently used oncomine database to determine the correlation between the NR2F2 level and the survival time. Although the survival rate had no significant difference (Fig. 1C), the high level of NR2F2 brought a shorter survival time than the low level of NR2F2 did. Then we used Kaplanmeier piotter database to determine the influence of NR2F2 on the survival of gastric cancer patients. The results told that the high level of NR2F2 caused poor clinical survival of patients. (Fig. 1D). All results conclude that the up-regulated NR2F2 level in gastric tissues is negatively correlated with patients' survival.

\section{Suppressive role of miR-27b in gastric cancer}

Enough evidence demonstrates that miR-27b, like miRNA, can suppress the proliferation of gastric cancer by targeting ROR1 (Tao et al., 2015). Currently, the function of miR-27b in gastric cancer metastasis remains unclear. To determine its exact function, Q-RT was performed to detect the expression level of miR-27b in gastric cancer tissues and cell lines. As is shown in Fig. 2A, miR-27b expression was significantly decreased in gastric cancer samples compared to matched normal tissues. Of all the patients, $89.47 \%$ (17/19) had a lower expression of miR-27b in tumor tissues than that in the adjacent mucosa (Fig. 2B). We further tested the correlation between the level of miR-27b expression and the metastasis of gastric cancer, finding that both were negatively associated (Fig. 2C). We also observed that miR-27b expression was lower in gastric cancer cell lines MGC-803 than in GES1 cells (human immortalized gastric epithelial cell line) (Fig. 2D). These results prove that the miR-27b level is down-regulated in gastric cancer tissues and gastric cancer cell lines, and is negatively correlated with the metastasis of gastric cancer.

\section{Cell proliferation, migration and invasion suppressed by $\mathrm{miR}-27 \mathrm{~b}$ in gastric cancer}

To evaluate the biological function of miR-27b in gastric cancer, the cell line MGC-803 with low expression of endogenous miR-27b was stably transfected with miR-27b by lentiviral infection (Fig. S2A). MTT assays showed that the ectopic overexpression of miR-27b suppressed the proliferation of MGC-803 cells (Fig. 3A). Moreover, soft agar formation assays revealed that overexpression miR-27b could inhibit the expanding of MGC-803 cellular colonies (Fig. 3B). The effects of miR-27b on the migration and invasion of gastric cancer cells were further examined by Transwell assays. The ectopic overexpression of miR-27b in MGC-803 cells dramatically inhibited cell migration (Fig. 3C). Also, we used a Matrigel transmembrane invasion assay to study the invasive properties of MGC-803 cells. Overexpression of miR-27b reduced the invasiveness of gastric cancer cells. Consistent with these phenotypes, then we detected that mRNA levels of MMP2, MMP9, Cyclin D1, c-Myc and the protein levels of c-Myc and Cyclin D1 were downregulated with overexpressed miR-27b (Fig. 3D and 3E). These results suggest that miR-27b can suppress the proliferation, migration and invasion of gastric cancer cells and inhibit the growth and metastasis of gastric cancer.

Proliferation, invasion and metastasis of GES-1 cells promoted by the knockdown of miR-27b Given that the overexpression of $\mathrm{miR}-27 \mathrm{~b}$ inhibited the proliferation, migration and invasion of gastric cancer cells, we performed loss-of-function experiments to verify the function of miR-27b in GES-1 cells. First, we used miR-27b inhibitor to knock down endogenous miR-27b in GES-1 cells. Q-RT detected that the level of miR-27b was significantly down-regulated by about $70 \%$ after the transfection with miR-27b inhibitor (Supplementary Figure 2B). Next, we explored whether miR27b inhibitor could augment the proliferation of GES-1 cells. Notably, the miR-27b inhibitor dramatically increased the ability of GES-1 cells in colony formation in a serial passage MTT assay and a soft agar assay (Fig. 4A and 4B). Similar data were obtained by Transwell analysis. The miR-27b 

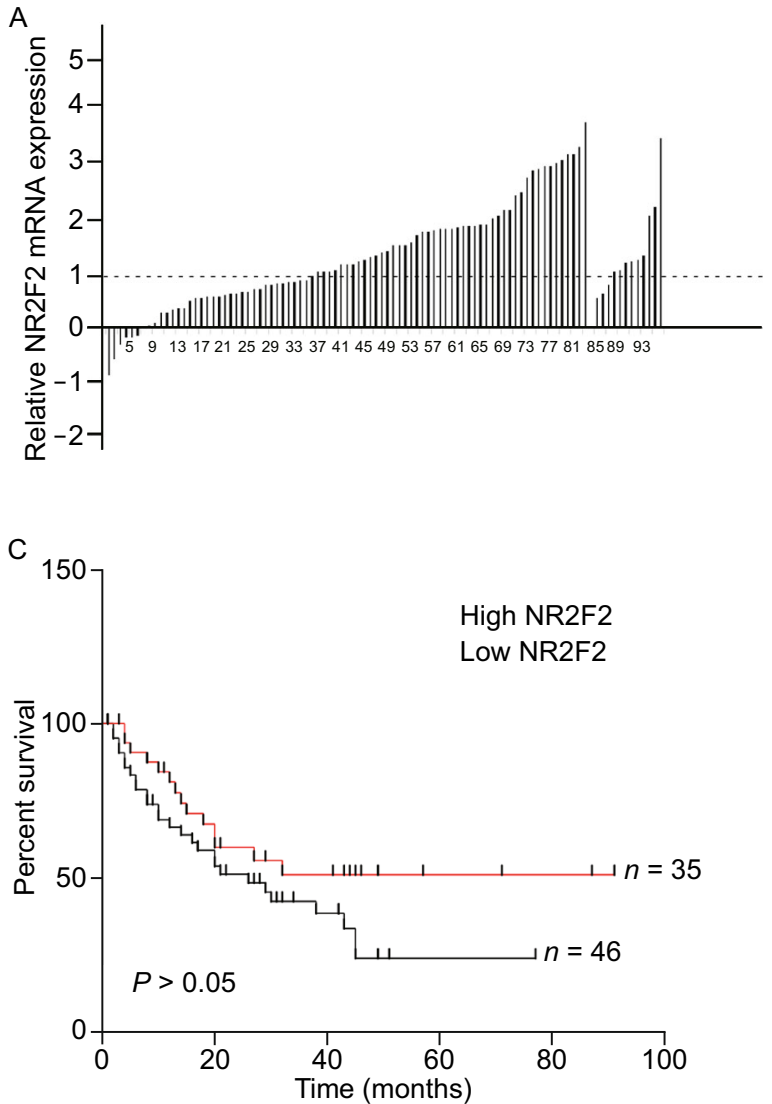

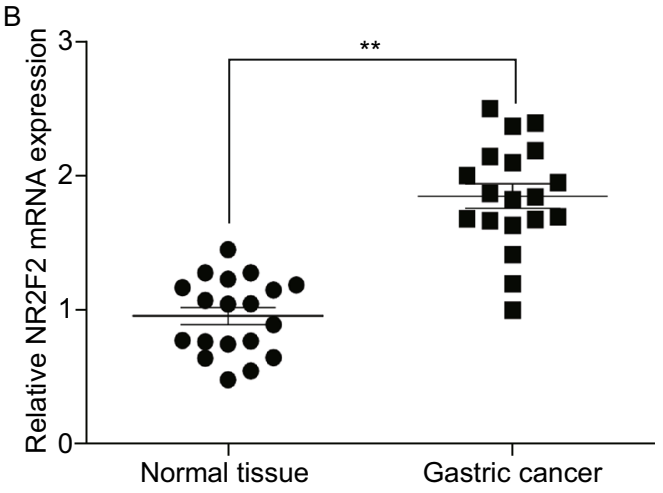

D

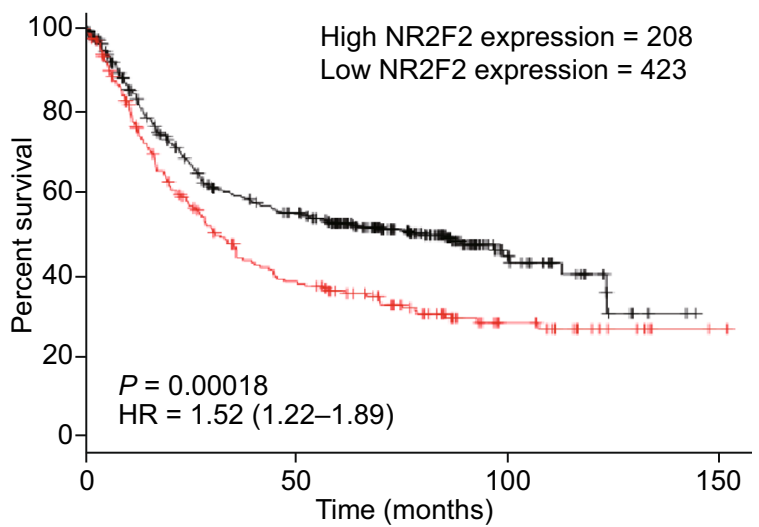

Figure 1. NR2F2 is up-regulated in human gastric cancer tissues and High NR2F2 level have poor clinical outcome. (A) The expression of NR2F2 in human gastric cancer tissue samples from oncomine database. (B) qRT-PCR analysis of NR2F2 expression in human gastric cancer tissue samples and their matched normal gastric tissues from 19 gastric cancer patients. (C) The High expression of NR2F2 in human gastric cancer tissues have trends to poor clinical outcome by oncomine database. (D) The High expression of NR2F2 in human gastric cancer tissues have poor clinical outcome by Kalpan meier-Poitter. Scale bars, $100 \mu \mathrm{m} .{ }^{*} P<$ $0.05,{ }^{* *} P<0.01,{ }^{* * *} P<0.001$.

increased the migration and invasion of GES-1 cells (Fig. 4C) and the mRNA expression of MMP2, MMP9 and CyclinD1, c-Myc. The protein levels of Cyclin D1 and C-Myc were dramatically upregulated (Fig. 4D and 4E).

Gastric tumor growth and metastasis inhibited by miR$27 \mathrm{~b}$ in vivo

To determine whether miR-27b can inhibit the growth and metastasis of gastric cancer cells in vivo, we generated luciferase-labeled MGC-803/miR-27b cells and their control counterparts, and then injected them into the orthotopic sites or spleens of nude mice. Compared with the mice injected with MGC-803/ctrl cells, the mice injected with a combination of MGC-803 with miR-27b cells displayed significantly smaller and lighter tumors four weeks later (Fig. 5A). Moreover, bioluminescence imaging of mice with a 4-week injection of MGC-803/miR-27b or MGC-803/ctrl cells showed that the metastasis of MGC-803 liver cells was significantly impaired by the ectopic overexpression of miR-27b (Fig. 5B).

\section{NR2F2 directly targeted by miR-27b}

To reveal the underlying mechanism in which miR-27b inhibits the growth and metastasis of gastric cancer cells, we used silico algorithms Targetscan, miRanda and some other methods to predict the target genes of miR-27b. We found NR2F2 with greater than $30 \%$ decreased expression upon ectopic miR-27b overexpression in MGC-803 cells and miR$27 \mathrm{~b}$ inhibitor increased the level of NR2F2 expression (Fig. 6A). We constructed luciferase reporter vectors containing wide-type or mutant 3'UTRs of NR2F2 (Fig.6B). Luciferase activity assays revealed that miR-27b suppressed the expression of luciferase containing 3'UTRs of NR2F2, compared with controls on 293T cells and MGC-803 cells (Fig. 6C). We found possible binding sites of miR-27b in 3'UTRs of NR2F2, and obliterated these sites by QuickChange PCR (Zheng et al., 2004). As is shown in Fig. 6D, the mutation of binding sites in 3 'UTRs of NR2F2 reversed the downregulation on luciferase activity induced by miR$27 \mathrm{~b}$, and abrogated the suppressing effect of miR-27b 
A
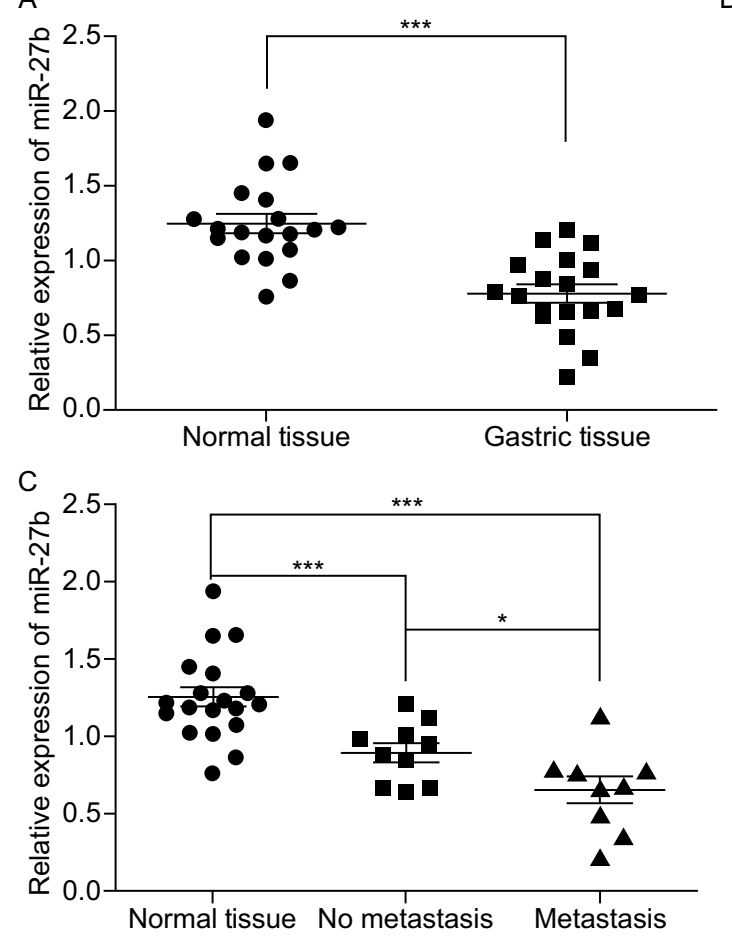

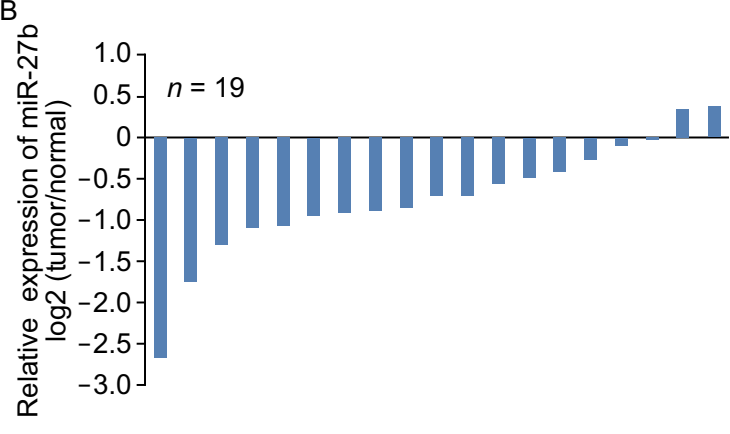

Figure 2. miR-27b is downregulated in human gastric cancer tissues and metastatic gastric cancer cell lines. (A) The expression of miR-27b in human gastric cancer tissue samples and matched normal tissues from 19 gastric cancer patients was analyzed by real-time PCR. (B) miR-27b were expressed as log2 fold change to show the relative expression in every paired sample. (C) Correlation between the lymph node metastasis status and the level of miR-27b in gastric cancers $(n=19)$. (D) The expression of $\mathrm{miR}-27 \mathrm{~b}$ in gastric cancer cell lines with different metastatic potentials was analyzed by real-time PCR. Scale bars, $100 \mu \mathrm{m}$. ${ }^{*} P<0.05$, ${ }^{* *} P<0.01,{ }^{* * *} P<0.001$.

overexpression. These results indicate that NR2F2 is the direct target of miR-27b in gastric cancer cells.

\section{DISCUSSION}

Tumorigenesis and metastasis are complex neoplastic processes triggered by a body of transcriptive factors. The abnormal expression of miRNA is involved in tumor proliferation and invasion by inhibiting their target genes (Zhang and Ma, 2012; Zhang et al., 2014b). Therefore, the identification of specific miRNAs and their targets engaged in tumorigenesis and metastasis can provide clues for diagnosing, treating and preventing the cancer. The mechanism that miR-27b participates in gastric cancer proliferation is clear (Tao et al., 2015), but the mechanism in metastasis remains elusive.. In this report, we demonstrated that miR$27 \mathrm{~b}$ took part in the proliferation and metastasis of gastric cancer. MiR-27b exhibited a decreased expression level in gastric cancer tissues. Moreover, in patients with gastric cancer, a correlation was clarified between lowered miR-27b expression and increased lymphatic metastasis. MiR-27b inhibited proliferation, migration, and invasion of gastric cancer cells in vitro and suppressed tumor growth and its metastasis to the liver in vivo. In conclusion, miR-27b is a tumor-suppressing gene in gastric cancer metastasis.

Nuclear receptor subfamily 2 (NR2F2, as known as COUPTFII) is found in many cancers, including breast cancer (Zhang et al., 2014a), ovarian cancer (Hawkins et al., 2013) and colorectal cancer (Zhou et al., 2016) . Oncomine database and Kaplan meier-piotter defined that NR2F2 was an oncogene in gastric cancer. With Targetscan, miRanda, mirwalk, and Pictar databases, we found highly conserved miR$27 \mathrm{~b}$ binding sites in the 3'UTRs of NR2F2. Moreover, the miR-27b level was inversely associated with the expression of NR2F2 in gastric cancer tissues, indicating that miR-27b can inhibit gastric cancerous proliferation and metastasis, at least in part, by downregulating the levels of NR2F2.

In all, this experiment provides evidence that miR-27b is a suppressor gene in gastric cancer proliferation and metastasis. MiR-27b can inhibit the proliferation and metastasis of gastric cancer cells by suppressing NR2F2. Furthermore, this experiment provides a potential diagnostic and prognostic marker for gastric cancer metastasis to liver. 
A

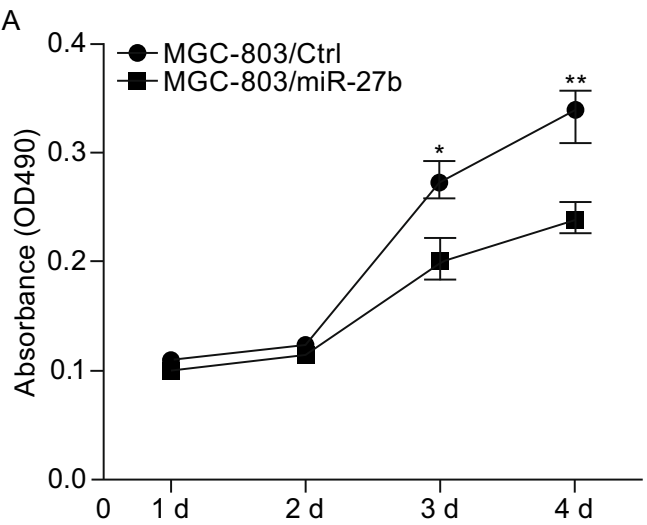

C

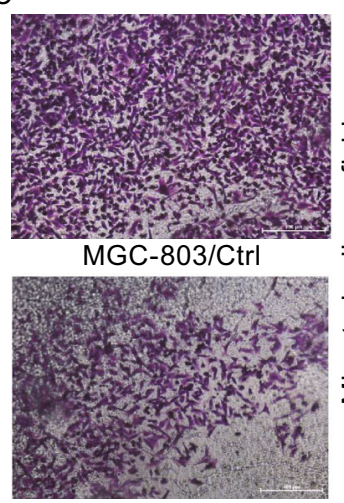

MGC-803/miR-27b

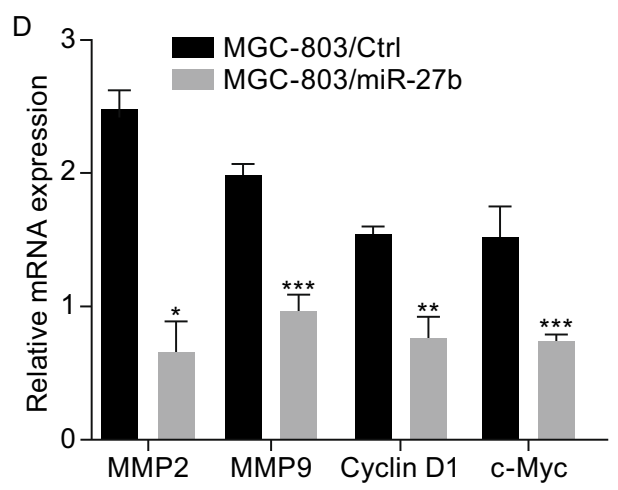

E
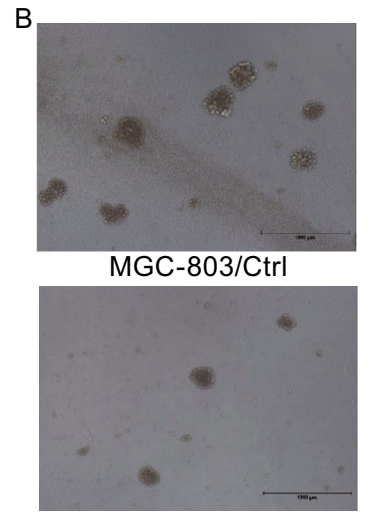

MGC-803/miR-27b
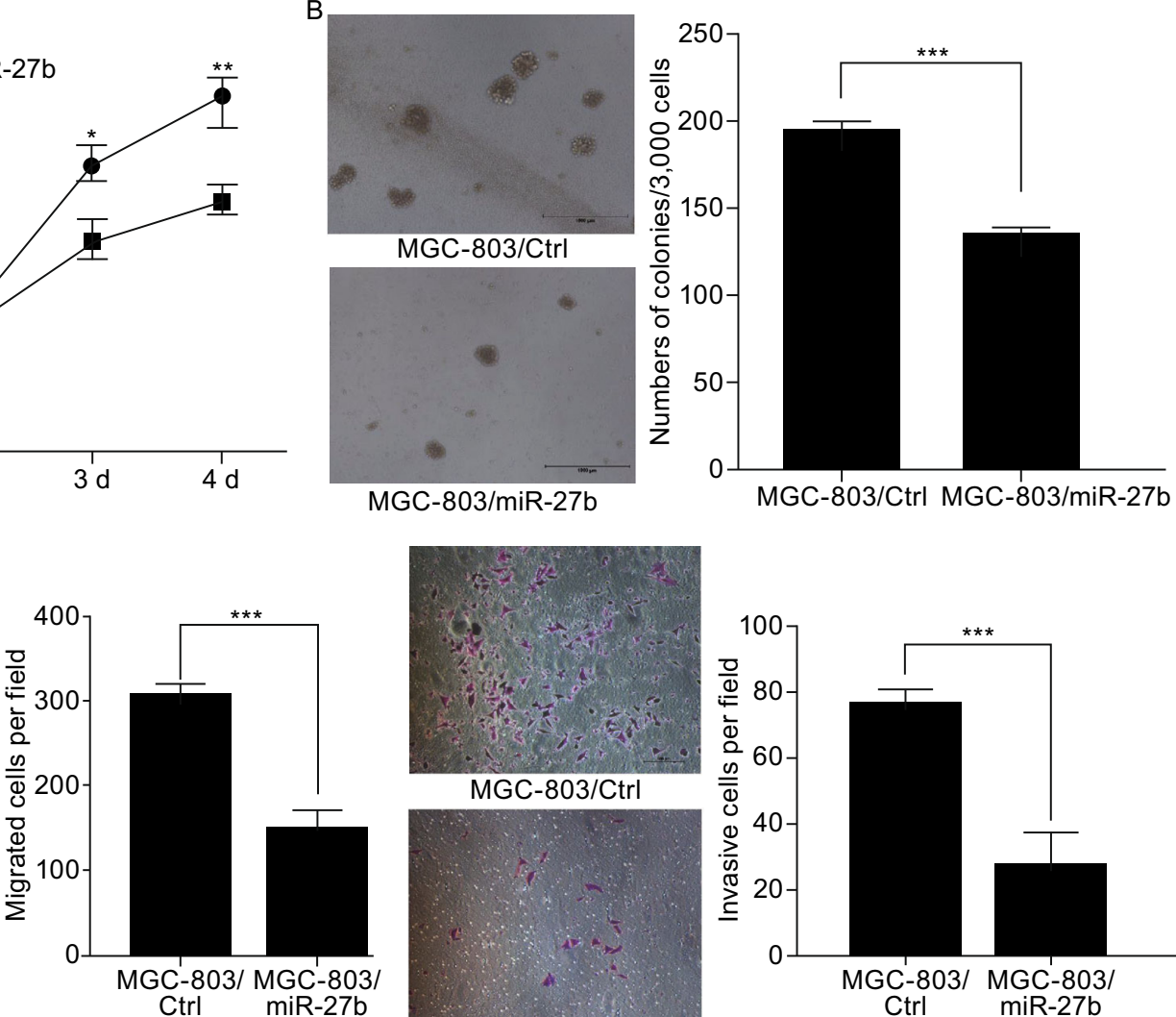

MGC-803/Ctrl
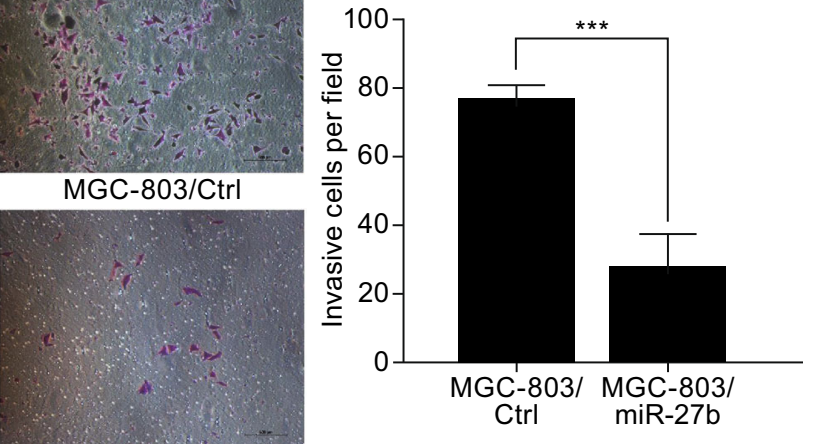

MGC-803/miR-27b

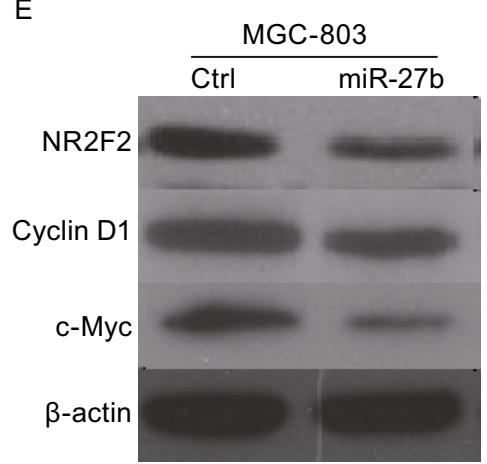

Figure 3. miR-27b inhibits gastric cancer cell growth, migration, and invasion in vitro. (A) The effect on cell proliferation of miR$27 \mathrm{~b}$ overexpression in MGC-803 cells was determined by the MTT assay. (B) Representative images show the colony formation of MGC-803/miR-27b and their control cells (left panel). Average colonies in each well for each group were counted from three independent experiments (right panel). (C) The effects of miR-27b overexpression in MGC-803 cells on cell migration and invasion were analyzed by Transwell migration and Matrigel-coated Transwell invasion analyses. (D) Q-RT analysis of proliferation and metastasis genes. (E) Western blot analysis of proliferation and metastasis genes. ${ }^{*} P<0.05,{ }^{* *} P<0.01,{ }^{* \star *} P<0.001$.

\section{MATERIALS AND METHODS}

Cell lines and clinical samples

GES-1, MGC-803, BGC-823, SGC-7901 and 293T cells were saved in our lab (from Institute of Health Sciences, Shanghai Institute for Biological Sciences, Shanghai). 293T cells were cultured in DMEM supplemented with $10 \%$ fetal bovine serum. GES-1, MGC-803,
BGC-823 and SGC-7901 cells were maintained in RPMI 1640 media supplemented with $10 \%$ fetal bovine serum.

Nineteen paired gastric cancer and normal adjacent tissues were collected from The Affiliated Drum Tower Hospital of Medical School of Nanjing University, Jiangsu, China. All patients were diagnosed pathologically according to the criteria of the American Joint Committee on Cancer by two professional pathologists independently. 


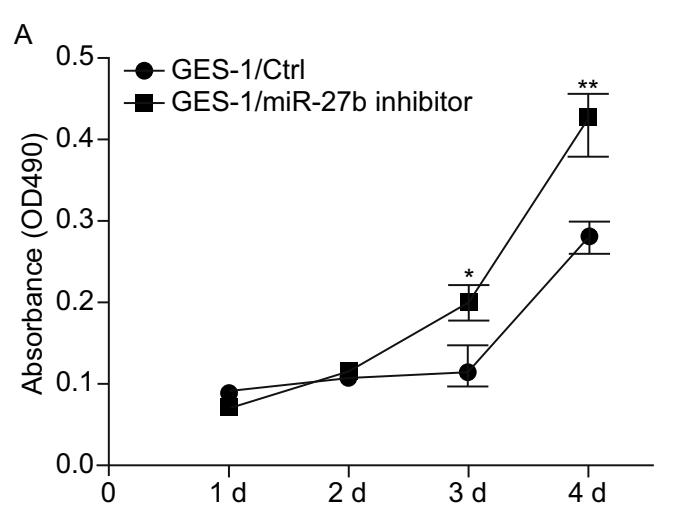

C
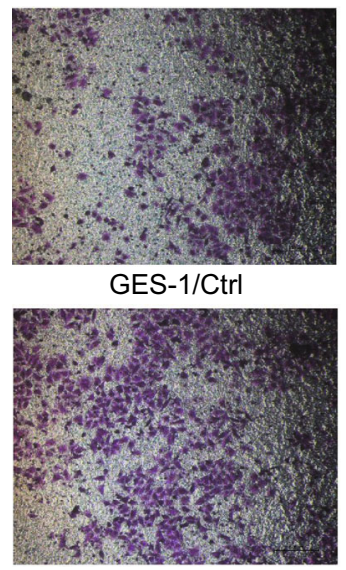

GES-1/miR-27b inhibitor

D

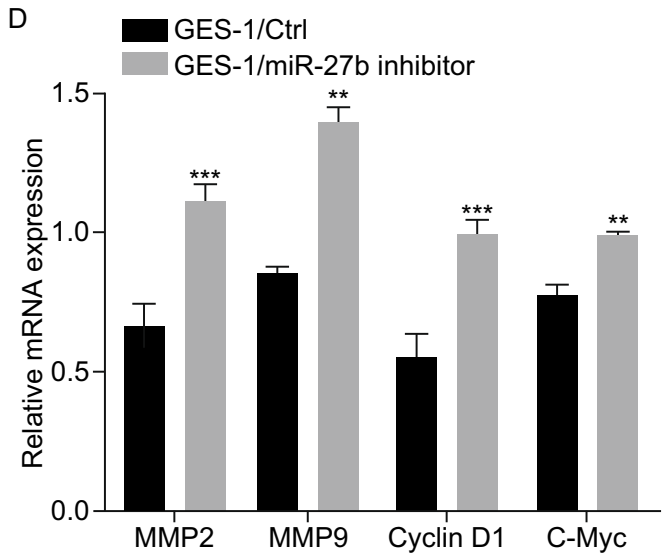

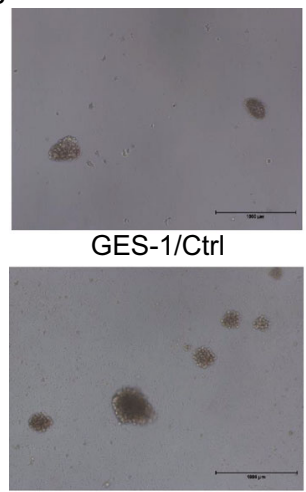
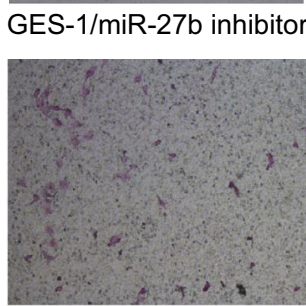

GES-1/Ctrl

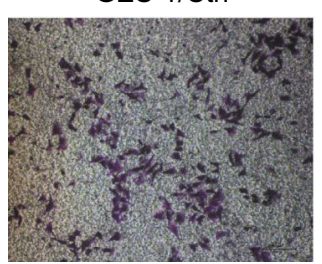

GES-1/miR-27b inhibitor

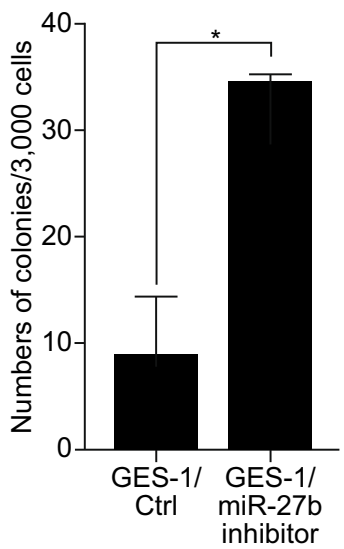

$E$
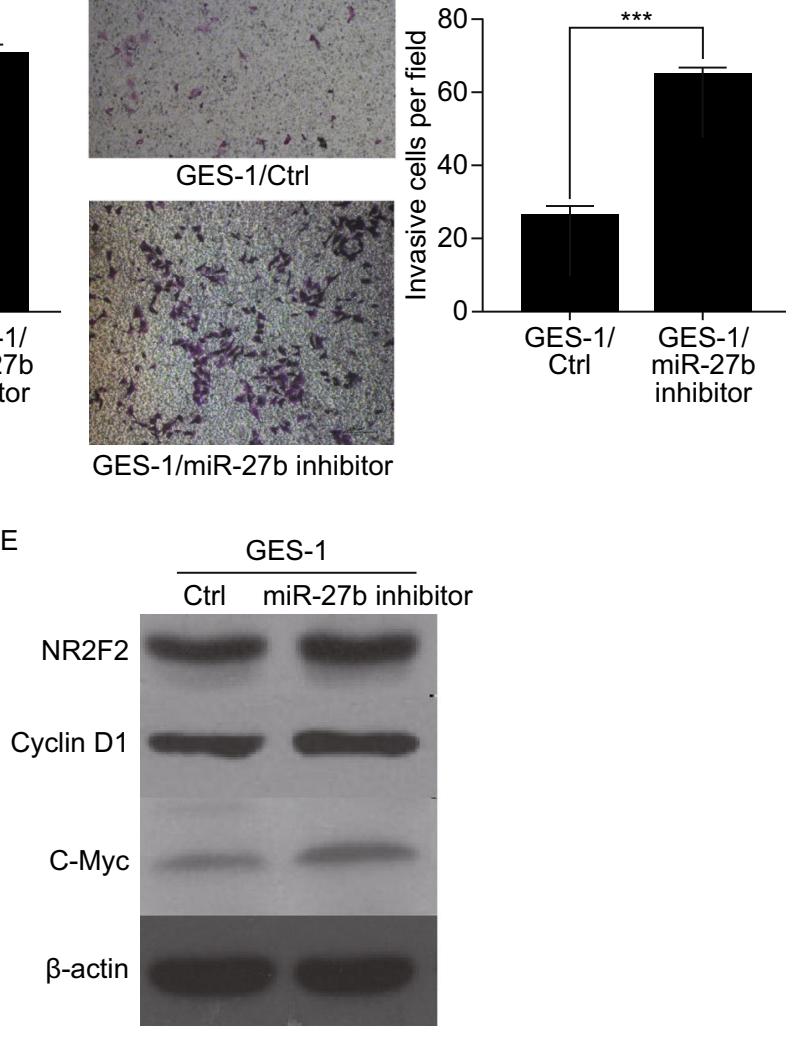

Figure 4. miR-27b inhibitor promotes gastric cancer cell growth, migration, and invasion in vitro. (A) The effect on cell proliferation of miR-27b inhibitor in GES-1 cells was determined by the MTT assay. (B) Representative images show the colony formation of GES-1/miR-27b inhibitor and their control cells (left panel). Average colonies in each well for each group were counted from three independent experiments (right panel). (C) The effects of miR-27b inhibitor in GES-1 cells on cell migration and invasion were analyzed by Transwell migration and Matrigel-coated Transwell invasion analyses. (D) Q-RT analysis proliferation and metastasis genes. (E) Western blot analysis of proliferation and metastasis genes. ${ }^{*} P<0.05,{ }^{* *} P<0.01,{ }^{* * *} P<0.001$.

Specimens were obtained with informed consent and the study was performed in accordance with the approved guidelines by the Medical Ethics Committee of the Affiliated Drum Tower Hospital of Nanjing University (Nanjing, China).

\section{Plasmid construction and generation of stable cell lines}

An hsa-miR-27b-containing flank region was amplified from human genomic DNA and inserted into pCDH-CMV-EF1-GFP+puro (System Biosciences). The entire lengths of the 3'UTRs of NR2F2 were 
A
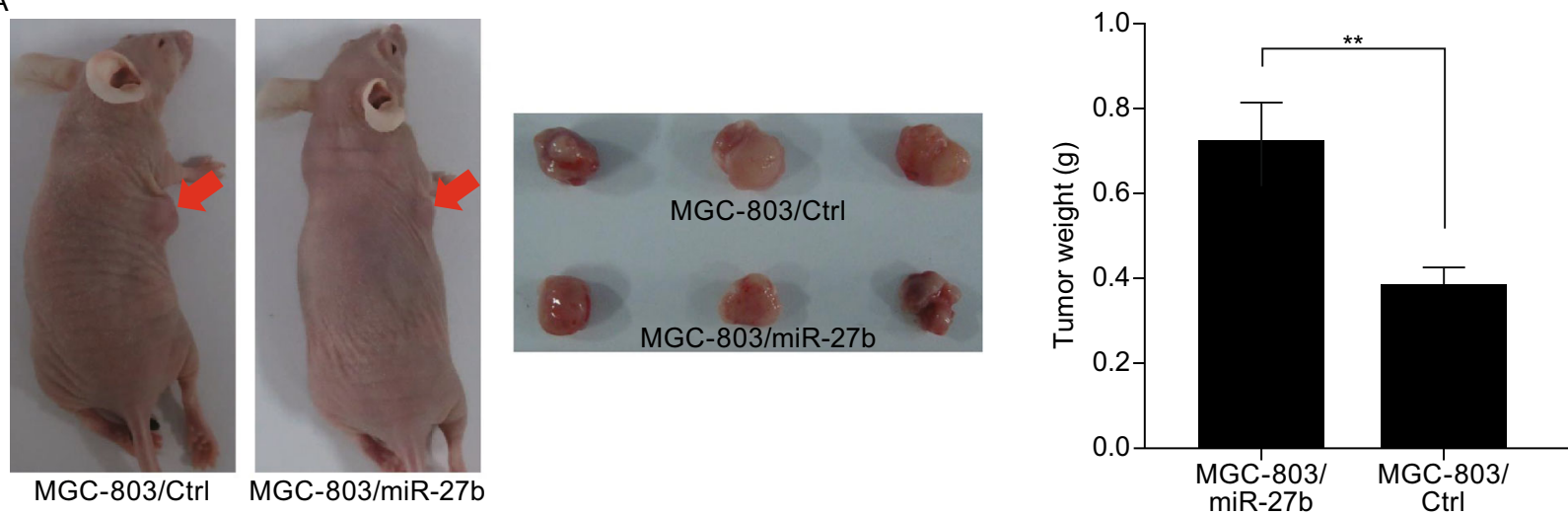

B
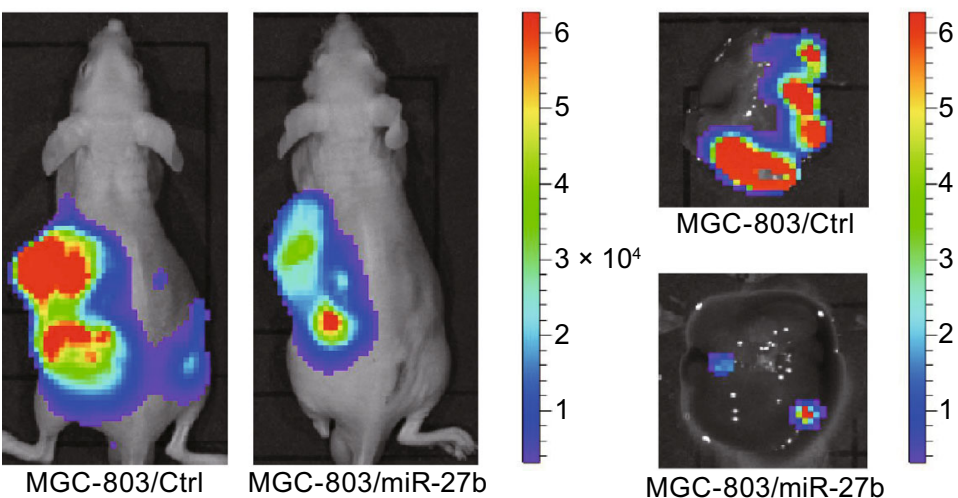

$3 \times 10^{4}$

MGC-803/Ctrl

MGC-803/Ctrl

MGC-803/miR-27b
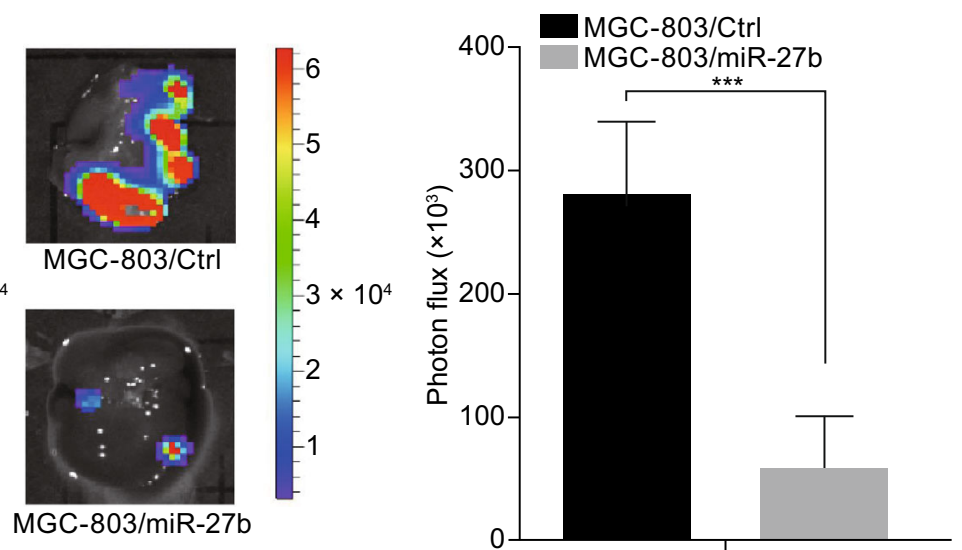

Liver metastasis

Figure 5. miR-27b suppresses tumor growth and liver metastasis of gastric cancer cells in vivo. (A) Representative images of primary tumors in the nude mice orthotopically injected with MGC-803/miR-27b or MGC-803/ctrl cells in the leg (left). Quantification of tumors in each group is shown (right). (B) Representative bioluminescence images of mice injected with MGC-803/miR-27b or MGC$803 /$ ctrl cells into spleen to show liver metastases (left). Quantification of liver metastases was analyzed by bioluminescence measurement (right). Scale bars, $100 \mu \mathrm{m} .{ }^{*} P<0.05,{ }^{* *} P<0.01,{ }^{* *} P<0.001$.

cloned into the pMIR-REPORT miRNA Expression Reporter Vector (Ambion). To generate a miR-27b-expressing stable cell line, a lentivirus-mediated packaging system containing four plasmids, pCDH-miR-27b or control plasmid, pMDL, REV, and VSVG, was used. To knock down miR-27b in GES-1 cells, miR-27b inhibitor was used GenePharma Colone Primer sequences were as follows: miR27b Fw-GC TCTAGA TTGCCAGGGATTACCACGCAA; Rv-CG GGATCC CTAGCATTCCCAGCAGGAGACAG NR2F2; 3'UTR FwCG ACGCGT AAGAAGGGGGAGTGAAACAGAG; Rv-CCC AAGCTT AGCAAGTTGTTCTGACCGACA.

\section{Western blot}

Western blot was performed as described previously (Lin et al., 2015). Cell lysates were separated on SDS-polyacrylamide gels and immunoblot analysis was performed with primary antibodies against NR2F2 (Abcam, Cambridge, MA, USA), $\beta$-actin (Millipore, Billerica, MA, USA), c-Myc (Cell Signaling Technology, Danvers, MA) and Cyclin D1 (Sigma-Aldrich).
Cell growth assay

For the MTT assay, 1000 cells were seeded and transfected in a 96-well plate. After transfection for 1, 2, 3, 4 days, and the absorbance at $490 \mathrm{~nm}$ was measured. For soft agar assay, 3000 cells were seeded and transfected in a 6-well plate. After transfection for 14 days, and the size and number of soft agar colonies were measured. Experiments were repeated three times.

Oncomine and TCGA database analysis

The Oncomine, TCGA and Kalpan meier-plotter database was searched for NR2F2 gene. The data sets containing expression data for NR2F2 were filtered to display up-regulation in gastric cancer versus normal tissue with $P<0.05$ and have poor clinical outcome.

Real-time quantitative PCR

Total RNA was extracted from the cultured cells or frozen tissues using TRIzol reagent (Invitrogen, Carlsbad, California, USA). The mRNA was 
A
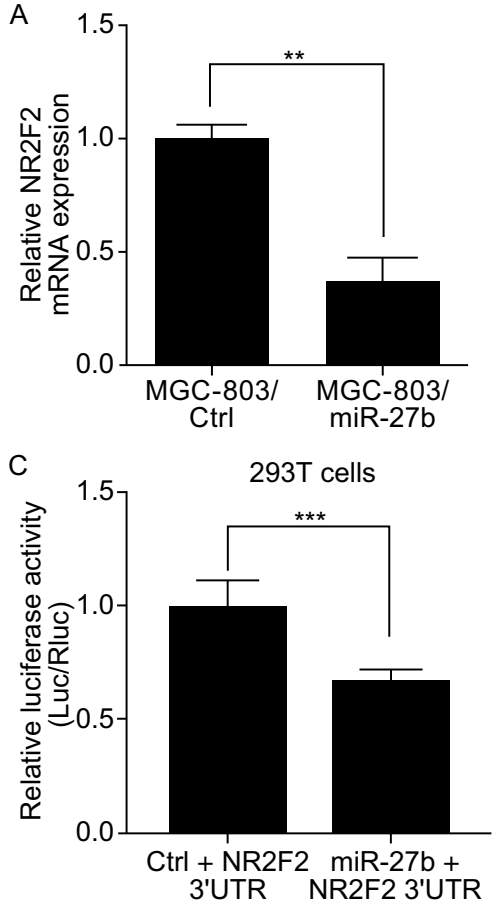
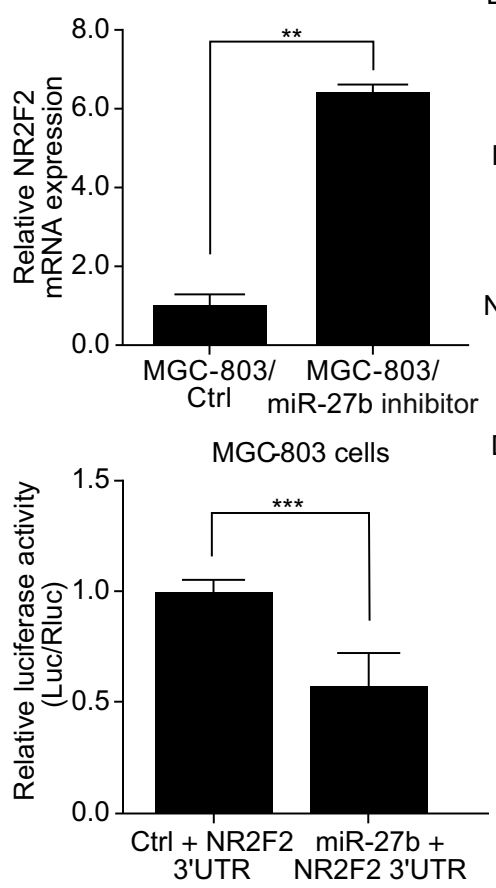

B
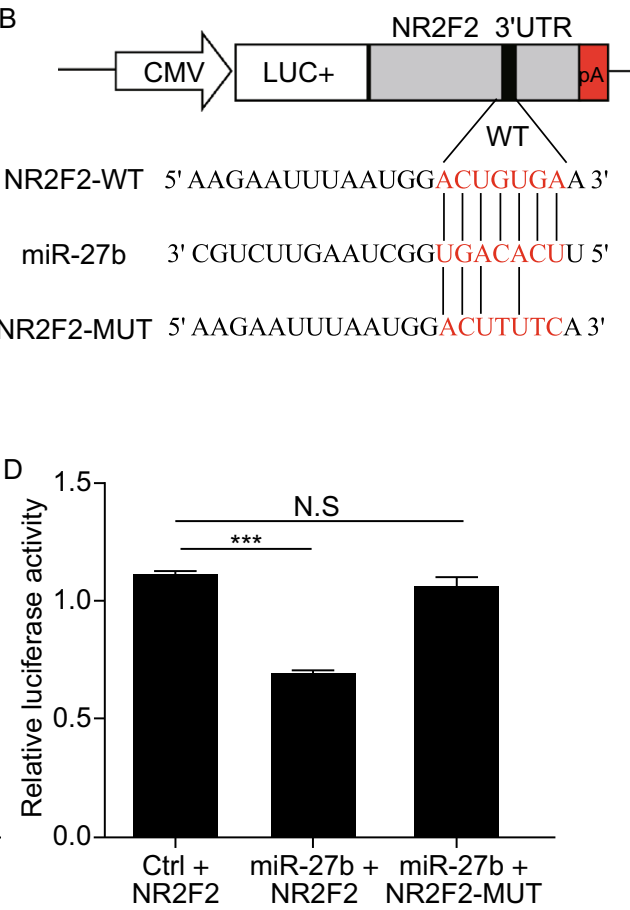

Figure 6. NR2F2 are direct targets of miR-27b. (A) The mRNA levels of NR2F2 in MGC-803/miR-27b and MGC-803/ctrl cells were analyzed by real-time PCR. The mRNA levels of NR2F2 in GES-1/miR-27b inhibitor and GES-1/ctrl cells were further analyzed by real-time PCR. (B) Constructed luciferase reporter vectors containing wild-type or mutant 3'UTRs of NR2F2. (C) The effects of miR$27 \mathrm{~b}$ overexpression on the activity of the 3'UTRs of target genes in 293T cells and MGC-803 cells were analyzed by the dual luciferase reporter assay. (D) The effects of miR-27b expression on the activity of wild-type and mutant 3'UTRs of NR2F2 were analyzed by the dual luciferase reporter assay. N.S.: no significance, ${ }^{\star} P<0.05$, ${ }^{\star \star} P<0.01,{ }^{* \star \star} P<0.001$.

reverse-transcribed by an RT-PCR kit (Invitrogen, Carlsbad, California, USA) according to the manufacturer's instructions. Quantitative PCR was then performed with primers for miR-27b, NR2F2 using SYBR Green PCR Master Mix (Invitrogen, Carlsbad, California, USA) in a real-time PCR System (Applied Biosystems, Carlsbad, California, USA) following a standard quantitative PCR procedure. Primer sequences used are shown in Table S1.

Relative quantification was performed by normalization to the amount of GAPDH or U6.

\section{Cell motility and invasion assay}

Migration and invasion assays were performed as described previously (Liu et al., 2014). All experiments were performed at least three times in triplicate.

\section{miRNA reporter luciferase assay}

For luciferase reporter assays, 293T cells and MGC-803 cells were seeded into a 24-well plate and co-transfected with 3 'UTR-luciferase and either miR-27b or control plasmids. Cells were harvested after two days and assayed using the Dual-Glo Luciferase Assay System (Promega) to determine the relative luciferase activity. The luciferase activity was measured by a luciferin enzyme detection assay kit
(Promega) and normalized to Renilla luciferase activity. Each treatment was performed in triplicate in three independent experiments.

\section{Animal studies}

All of the experiments using animals were performed in accordance with a protocol approved by the Animal Care and Use Committee of Nanjing University. For tumor growth assays, $5 \times 10^{6}$ cells were subcutaneously injected into the lower back regions of 6 -week-old male nude mice for four weeks ( $n=3$ per group). For orthotropic metastasis, 6-week-old male nude mice were anesthetized and their spleens were exteriorized by laparotomy, and then $5 \times 10^{5}$ cells were injected into the spleens for four weeks ( $n=3$ per group). Tumor growth and hepatic metastases at day 28 were monitored using the live animal Lumina II system (Xenogen IVIS system).

\section{Statistical analysis}

All data were expressed as the mean $\pm S D$. Statistical analysis was performed with Student's $t$-test. A $P$ value less than 0.05 was considered statistically significant.

\section{ACKNOWLEDGEMENTS}

This work supported by the Fundamental Research Funds for the Central Universities (No. 021414380015, 021414380155). 


\section{COMPLIANCE WITH ETHICS GUIDELINES}

Qingzhao Feng, Xionglin Wu, Fuchao Li, Beibei Ning, Xiaofeng Lu, Yin Zhang, Ying Pan and Wenxian Guan declare that they have no conflict of interest.

All procedures followed were in accordance with the ethical standards of the responsible committee on human experimentation (institutional and national) and with the Helsinki Declaration of 1975, as revised in 2000 (5). Informed consent was obtained from all patients for being included in the study. All institutional and national guidelines for the care and use of laboratory animals were followed.

\section{OPEN ACCESS}

This article is distributed under the terms of the Creative Commons Attribution 4.0 International License (http://creativecommons.org/ licenses/by/4.0/), which permits unrestricted use, distribution, and reproduction in any medium, provided you give appropriate credit to the original author(s) and the source, provide a link to the Creative Commons license, and indicate if changes were made.

\section{REFERENCES}

Aleckovic M, Kang Y (2015) Regulation of cancer metastasis by cellfree miRNAs. Biochim Biophys Acta 1855:24-42

Hawkins SM, Loomans HA, Wan YW, Ghosh-Choudhury T, Coffey D, Xiao W, Liu Z, Sangi-Haghpeykar H, Anderson ML (2013) Expression and functional pathway analysis of nuclear receptor NR2F2 in ovarian cancer. J Clin Endocrinol Metab 98:E1152E1162

Huang CY, Chang AC, Chen HT, Wang SW, Lo YS, Tang CH (2016) Adiponectin promotes VEGF-C-dependent lymphangiogenesis by inhibiting miR-27b through CaMKII/AMPK/p38 signaling pathway in human chondrosarcoma cells. Clin Sci 130(17):15231533

Kang W, Tong JH, Chan AW, Zhao J, Dong Y, Wang S, Yang W, Sin $\mathrm{FM}, \mathrm{Ng} \mathrm{SS}, \mathrm{Yu} \mathrm{J}$ et al (2014) Yin Yang 1 contributes to gastric carcinogenesis and its nuclear expression correlates with shorter survival in patients with early stage gastric adenocarcinoma. J Trans Med 12:80

Kieback DG, Levi T, Kohlberger P, Fiedrich U, Press MF, Rosenthal HE, Mobus VJ, Runnebaum IB, Tong XW, Tsai MJ (1996) Chicken ovalbumin upstream promoter-transcription factor (COUP-TF) expression in human endometrial cancer cell lines. Anticancer Res 16:3371-3376

Lee JJ, Drakaki A, lliopoulos D, Struhl K (2012) MiR-27b targets PPARgamma to inhibit growth, tumor progression and the inflammatory response in neuroblastoma cells. Oncogene 31:3818-3825

Lin Y, Liu AY, Fan C, Zheng H, Li Y, Zhang C, Wu S, Yu D, Huang Z, Liu $F$ et al (2015) MicroRNA-33b inhibits breast cancer metastasis by targeting HMGA2, SALL4 and Twist1. Sci Rep 5:9995
Liu AY, Cai Y, Mao Y, Lin Y, Zheng H, Wu T, Huang Y, Fang X, Lin S, Feng $Q$ et al (2014) Twist2 promotes self-renewal of liver cancer stem-like cells by regulating CD24. Carcinogenesis 35:537-545

Ma L, Teruya-Feldstein J, Weinberg RA (2007) Tumour invasion and metastasis initiated by microRNA-10b in breast cancer. Nature 449:682-688

Milne AN, Carneiro F, O'Morain C, Offerhaus GJ (2009) Nature meets nurture: molecular genetics of gastric cancer. Human Genet 126:615-628

Prahalad P, Dakshanamurthy S, Ressom H, Byers SW (2010) Retinoic acid mediates regulation of network formation by COUPTFIl and VE-cadherin expression by TGFbeta receptor kinase in breast cancer cells. PloS ONE 5:e10023

Qin J, Chen X, Yu-Lee LY, Tsai MJ, Tsai SY (2010) Nuclear receptor COUP-TFII controls pancreatic islet tumor angiogenesis by regulating vascular endothelial growth factor/vascular endothelial growth factor receptor-2 signaling. Cancer Res 70:8812-8821

Qin J, Wu SP, Creighton CJ, Dai F, Xie X, Cheng CM, Frolov A, Ayala G, Lin X, Feng XH et al (2013) COUP-TFII inhibits TGFbeta-induced growth barrier to promote prostate tumorigenesis. Nature 493:236-240

Ruan K, Fang X, Ouyang G (2009) MicroRNAs: novel regulators in the hallmarks of human cancer. Cancer Lett 285:116-126

Shin SW, Kwon HC, Rho MS, Choi HJ, Kwak JY, Park JI (2009) Clinical significance of chicken ovalbumin upstream promotertranscription factor II expression in human colorectal cancer. Oncol Rep 21:101-106

Tao J, Zhi X, Zhang X, Fu M, Huang H, Fan Y, Guan W, Zou C (2015) miR-27b-3p suppresses cell proliferation through targeting receptor tyrosine kinase like orphan receptor 1 in gastric cancer. J Exp Clin Cancer Res 34:139

Tsai SY, Tsai MJ (1997) Chick ovalbumin upstream promoter-transcription factors (COUP-TFs): coming of age. Endocr Rev 18:229-240

Yan Y, Wang LF, Wang RF (2015) Role of cancer-associated fibroblasts in invasion and metastasis of gastric cancer. World J Gastroenterol 21:9717-9726

Yao J, Deng B, Zheng L, Dou L, Guo Y, Guo K (2016) miR-27b is upregulated in cervical carcinogenesis and promotes cell growth and invasion by regulating $\mathrm{CDH} 11$ and epithelial-mesenchymal transition. Oncol Rep 35:1645-1651

Zhang J, Ma L (2012) MicroRNA control of epithelial-mesenchymal transition and metastasis. Cancer Metastasis Rev 31:653-662

Zhang C, Han Y, Huang H, Qu L, Shou C (2014a) High NR2F2 transcript level is associated with increased survival and its expression inhibits TGF-beta-dependent epithelial-mesenchymal transition in breast cancer. Breast Cancer Res Treat 147:265-281

Zhang Y, Yang P, Wang XF (2014b) Microenvironmental regulation of cancer metastasis by miRNAs. Trends Cell Biol 24:153-160

Zheng L, Baumann U, Reymond JL (2004) An efficient one-step sitedirected and site-saturation mutagenesis protocol. Nucleic Acids Res 32:e115

Zhou B, Song J, Han T, Huang M, Jiang H, Qiao H, Shi J, Wang $Y$ (2016) MiR-382 inhibits cell growth and invasion by targeting NR2F2 in colorectal cancer. Mol Carcinog. doi:10.1002/mc.22466 